Marquette University

e-Publications@Marquette

College of Nursing Faculty Research and

Publications

Nursing, College of

3-2007

\title{
Efficacy of Cervical Mucus Observations Plus Electronic Hormonal Fertility Monitoring as a Method of Natural Family Planning
}

Richard Fehring

Marquette University, richard.fehring@marquette.edu

Mary Schneider

Marquette University, mary.schneider@marquette.edu

Kathleen Raviele

Mary L. Barron

St Louis University

Follow this and additional works at: https://epublications.marquette.edu/nursing_fac

Part of the Nursing Commons

\section{Recommended Citation}

Fehring, Richard; Schneider, Mary; Raviele, Kathleen; and Barron, Mary L., "Efficacy of Cervical Mucus Observations Plus Electronic Hormonal Fertility Monitoring as a Method of Natural Family Planning" (2007). College of Nursing Faculty Research and Publications. 10.

https://epublications.marquette.edu/nursing_fac/10 


\section{Efficacy of Cervical Mucus Observations Plus Electronic Hormonal Fertility Monitoring as a Method of Natural Family Planning}

Authors: Richard J. Fehring, Mary Schneider, Kathleen Raviele, Mary Lee Barron

Abstract:

Objective: To determine the effectiveness of an electronic hormonal fertility monitor plus cervical mucus monitoring to avoid pregnancy.

Design: A 12-month prospective clinical efficacy trial.

Setting and Participants: One hundred ninety-five (195) women (mean age 29.8 years) seeking to avoid pregnancy with a natural method at 5 clinical sites in 4 cities.

Intervention: Each participant was taught to track fertility by self-observation of cervical mucus and an electronic monitor that measures urinary levels of estrone-3-glucuronide and luteinizing hormone.

Main Outcome Measures: Correct- and typical-use unintended pregnancy rates.

Results: There were a total of 26 unintended pregnancies, 3 with correct use. With 1,795 months of use, the correct-use pregnancy rate was 2.1\% per 12 months of use (i.e., $97.9 \%$ effective in avoiding pregnancy when rules of the method were always followed) and the imperfect-use pregnancy rate was $14.2 \%$ per 12 months of use (i.e., $85.8 \%$ effective in avoiding pregnancy when rules of the method were not always followed and all unintended pregnancies and months of use were included in the calculations).

Conclusions: Correct use of an electronic hormonal fertility monitor with cervical mucus observations can be as effective as other fertility awareness - based methods of natural family planning. Comparative studies are needed to confirm this conclusion.

Approximately 124,000 women in the United States use natural methods of family planning (i.e., cervical mucus or temperature monitoring) for avoiding pregnancy (Mosher, Martinez, Chandra, Abma, \& Wilson, 2004). Another 434,000 use self-devised calendar formulas (i.e., rhythm) as a means to avoid pregnancy. Many women rely on natural markers of fertility to help them achieve pregnancy. The accuracy, ease of use, acceptability, and effectiveness of natural biological markers to estimate the time of fertility in the menstrual cycle is important for these women.

1 Fehring, Schneider, Raviele, \& Barron 
There have been few new natural methods of family planning in the past 40 years (Fehring, 2005). Furthermore, the use of natural family planning (NFP) has not increased over the past 20 years (Mosher et al., 2004). Current methods of NFP rely on calendar calculations, changes in cervical vaginal fluid observations, basal body temperature (BBT) shifts, and any combination of these natural signs of fertility. Many systems of NFP have evolved, based on these natural biological signs, with different types of charting methods, teaching pedagogy, follow-up frequency, and rules for avoiding and achieving pregnancy.

When NFP methods are used according to instructions (which is referred to as correct use), the unintended pregnancy rate can be as low as 1 to 3 per 100 users over a 12-month time period, and when the instructions are not always followed (which is called imperfect use), the unintended pregnancy rate can be as high as 25 per 100 users over 12 months (Trussell, 2004). The large difference between correct- and imperfect-use unintended pregnancy rates is due to the considerable overestimation of the 6-day fertile window (when correct use is calculated) and to difficulties in the complexity of learning and using NFP methods, the length of required abstinence, subjectivity of the biological markers to estimate the fertile window, and variability of the menstrual cycle (Burkman, 2002 ; Trussell).

The purpose of this study was to determine the effectiveness of using a new high-tech hormonal fertility monitor called the Clearblue Easy Fertility Monitor (CEFM) (Inverness Medical Innovations, Waltham, MA) along with another natural marker of fertility (i.e., cervical mucus monitoring) as a means to avoiding pregnancy. The CEFM is a handheld home-use device that estimates fertility by measuring urinary metabolites of estrogen and luteinizing hormone (LH) (May, 2001). The monitor is intended as an aid for achieving pregnancy. However, it could be useful for women and couples who wish to avoid pregnancy as well.

\section{Background Studies}

Researchers have demonstrated that there is essentially a 6-day window of fertility in a woman' $s$ menstrual cycle and that peak fertility occurs on the 2 days preceding ovulation (Dunson, Baird, Wilcox, \& Weinberg, 1999; Wilcox, Weinberg, \& Baird, 1995). The fertile window includes the 5 days before ovulation and the day of ovulation. The accurate determination of those 6 days of fertility (i.e., the fertile window) and the peak of fertility is critical for women and couples who wish to use natural biological markers to achieve or avoid pregnancy. The most common self-detected natural indicators for estimating the fertile phase of the menstrual cycle in use today are calendar-based formulas, daily measurement of BBT, selfobservation of cervical-vaginal mucus, and self-testing of $\mathrm{LH}$ in the urine. Each of these

\section{Fehring, Schneider, Raviele, \& Barron}


methods has advantages and disadvantages.

Calendar systems of family planning are simple to learn and require users only to monitor the length of their menstrual cycles and follow simple fertility formulas to determine the beginning and end of the fertile phase. An example calendar formula is to subtract 19 days from the shortest of the last 8 to 12 menstrual cycles to determine the beginning day of infertility and subtract the shortest from the longest of the last 8 to 12 menstrual cycles and add 8 days to that number to obtain the length and end of the fertile phase. To avoid pregnancy, couples abstain from genital sexual relations during the fertile time.

Kambic and Lamprecht (1996) conducted a meta-analysis of eight effectiveness studies of the calendar rhythm method and estimated an unintended pregnancy rate of 15.0 to 18.0 per 100 women with 12 months of observation. They concluded that these results are in the effectiveness range of barrier contraception. A problem with calendar-based systems of NFP is that the estimated fertile phase (and resulting abstinence) becomes very long when menstrual cycle lengths vary more than 7 days.

Basal body temperature recordings have been widely used to estimate ovulation for the purpose of avoiding and achieving pregnancy. Basal body temperature is based upon a postovulatory rise (or shift) in body temperature due to an increase in the hormone progesterone (Barron \& Fehring, 2005). While BBT is inexpensive and easy to use, it is often an imprecise predictor or indicator of ovulation (Guida et al., 1999; McCarthy \& Rockette, 1983; Quagliarello \& Arny, 1986). Body temperature can also rise due to other phenomena such as stress, disrupted sleep patterns, and infection. Basal body temperature is an incomplete measure of the fertile window in that it tells the user only the potential peak and end of the fertile window.

When BBT is used with another natural marker of fertility (such as a calendar formula and/or cervical mucus monitoring) to estimate the beginning of the fertile window, it can be an extremely effective means of NFP. Researchers have demonstrated a very low typical-use unintended pregnancy rate of 2.6 per 100 women over 13 cycles of use when BBT monitoring is used in combination with a simple calendar formula and cervical mucus monitoring as a double check to estimate the beginning and end of the fertile phase (Frank-Herrmann, Gnoth, Baure, Strowitski, \& Freundl, 2005). The problem with using several markers of fertility is that the method becomes more complex, the indicators at times are contradictory, and you multiply the disadvantages of each indicator of fertility. Other simple means of monitoring fertility have been developed to help women to predict and confirm the time of ovulation.

\section{Fehring, Schneider, Raviele, \& Barron}


The self-observation of cervical mucus has been used by many women to predict the beginning, peak, and end of their fertile phase. Determining optimal or peak cervical mucus, unlike BBT, is based on a preovulatory and estrogenic methodology. Estrogen, which stimulates the production of cervical mucus, rises in the serum before ovulation takes place. Optimal or "peak " cervical mucus (that stretches an inch or more is clear in appearance and has the sensation of lubrication) correlates $95 \%$ of the time within \pm 3 days of the estimated day of ovulation as determined by serum hormonal changes or serial ultrasound observation of the dominant follicle (Fehring, 2002).

In the late 1970s, a World Health Organization (WHO) Task Force conducted a fivecountry study to evaluate the effectiveness of a mucus-only observation method of NFP called the "ovulation method" (WHO Task Force, 1981). The correct-use unintended pregnancy rate was around 3.4 per 100 women users, but the total unintended pregnancy rate was closer to 22.5 per 100 women users over 12 months of use (Trussell \& Grummer-Strawn, 1991). Howard and Stanford (1999) reported an effectiveness study of a standardized cervical mucus monitoring method called the Creighton Model (CrM) with 701 couples who had a total of 17.12 pregnancies per 100 couples over a 12-month period. In an earlier effectiveness study of the $\mathrm{CrM}$ at Marquette University with 242 couples using the CrM, a correct-use 12-month unintended pregnancy rate of 2.0 and a use-effectiveness rate of 15.2 were found (Fehring, Lawrence, \& Philpot, 1994).

A problem with observing cervical mucus is that many women experience continuous and confusing mucus patterns and find it difficult to distinguish the actual fertile phase. On average, there are 15 days of mucus during the menstrual cycle (Colombo, Mion, Passarin, \& Scarpa, 2006). Furthermore, up to $17 \%$ of menstrual cycles will not have a peak day of cervical mucus (Colombo et al., 2006). Although most women with a continuous discharge can eventually determine their peak, this takes a considerable amount of time, patience, and the assistance of a knowledgeable practitioner or teacher - a particularly frustrating experience for women/couples who are trying to achieve or avoid pregnancy. A simple, objective, and accurate test to predict and detect ovulation would greatly help women with continuous cervical mucus as well as increase the effectiveness of natural methods of fertility control and awareness.

Detecting the LH surge in the urine is considered by experts to be a standard method and one of the best tests for indirectly self-predicting ovulation (Crosignani \& Rubin, 2000; Guida et al., 1999). Ovulation detection kits have been developed employing monoclonal antibody technology to detect the LH surge that occurs in the urine 12 to 24 hours before

\section{Fehring, Schneider, Raviele, \& Barron}


ovulation (Seibel, 1986). The manufacturers of ovulation test kits claim greater than $99 \%$ accuracy in detecting the LH surge. However, the test kits miss the LH surge or do not detect the surge in about 10\% of cycles (Fehring, 1990; Fehring, Raviele, \& Schneider, 2004; Nulsen, Wheeler, Ausmanas, \& Blasco, 1987). Furthermore, the LH surge in the urine does not give a long enough warning period before ovulation takes place in order to be used for avoiding pregnancy and frequently misses the two most fertile days in the cycle (Stanford, White, \& Hataska, 2002). Since test kits must be purchased for each menstrual cycle, there is an ongoing monthly expense. However, use of urinary LH detection has potential for use as a marker for fertility with NFP methods (Martinez, 1997; Martinez, Zinaman, Jennings, \& Lamprecht, 1995).

The CEFM is a small handheld electronic device that is designed to detect urinary metabolites of LH and estrogen (i.e., estrone-3-glucuronide [E3G]) from early-morning urine samples. The monitor reads the changing color levels of disposable test strips that have antibodies specific to E3G and LH impregnated into their latex wick-type structure. A benefit of the monitor is an objective reading of the test strips for the user. The monitor targets the optimal days to achieve a pregnancy and indicates three levels of fertility, that is, "low," "high," and "peak." The CEFM has a small LCD window screen that tells the user her daily fertility status, the day of her cycle, and whether or not a urine test is needed (see Figure 1).

European researchers collaborated on a study to determine the effectiveness of an electronic fertility monitor similar to the CEFM called Persona (Bonnar et al., 1999). The prototype version of Persona has the same threshold level of E3G and LH as the CEFM and utilizes the same type of test strip and inferred light reading system. The Persona study involved 710 women volunteers who were asked to use the fertility monitor (without any formal training) for the purpose of avoiding pregnancy for a 1year period. At completion of the study, there were 67 correct-use unintended pregnancies (i.e., a pregnancy resulted from having intercourse on a day that the monitor said was infertile), 92 user-related pregnancies (intercourse on days the monitor said the woman user was fertile), and 3 pregnancies of uncertain timing. A 13-cycle life table analysis yielded a correct-use pregnancy rate of $12.0 \%$.

Researchers conducted a study to determine the accuracy of the CEFM with 53 women volunteers and found (with transvaginal ultrasound to ascertain the precise day of ovulation) that ovulation occurred $97.0 \%$ of the time during a 3-day period that included the 2 peak days plus the next day high on the CEFM (Behre et al., 2000). There were no ultrasound-detected ovulations before the monitor peak days. Another study with 30 healthy women volunteers showed that a Japanese version of the CEFM called the Clearview Primera Fertility Monitor

\section{Fehring, Schneider, Raviele, \& Barron}


indicated up to 5 days of high fertility readings in $58.6 \%$ of the cycles (i.e., 17 out of 39 cycles) before the CEFM peak reading and in $92.8 \%$ of the cycles (i.e., 24 of 29 cycles) in a laboratorydetermined urinary LH peak (Tanabe et al., 2001). The authors of this study concluded that the device will allow couples to use the information to time intercourse for the best prospects of achieving pregnancy. A recent randomized control trial among couples trying to conceive for up to 2 years showed an increased likelihood of achieving pregnancy with users of the CEFM compared to a control group of nonuse (Robinson, Wakelin, \& Ellis, in 2007).

The information provided by the CEFM could be used inversely as an aid for avoiding pregnancy along with another check for the beginning (and sometimes end) of the fertile window. Once the monitor provides the 2 peak day readings followed by a day of high, there (theoretically) should be less than a $3 \%$ chance of an unintended pregnancy if intercourse is reserved for the post peak plus 3 full days. However, the monitor provides a 5-day warning (of high readings) before the first peak day only in about $20 \%$ of the cycles monitored (Fehring et al., 2004). Therefore, in approximately $80 \%$ of the cycles, the monitor will miss the beginning of the theoretical 6-day fertile window. So another marker of fertility is needed as a double check for the beginning of the fertile phase. When used in tandem with another biological marker of fertility (e.g., cervical mucus observations) as a double check for the beginning and end of the fertile phase, the high-tech monitor could be a very simple and effective means of fertility monitoring and avoiding pregnancy. The specific aim of this study was to evaluate the effectiveness of the CEFM plus cervical mucus monitoring to avoid pregnancy over a 12-month time period.

\section{Methods}

\section{Design}

This study was a 12-month prospective clinical trial of an electronic hormonal fertility monitor plus cervical mucus monitoring method to avoid pregnancy. The purpose of the trial was to provide preliminary evidence for effectiveness in avoiding pregnancy.

\section{Sample}

Couples seeking to learn how to avoid pregnancy by use of a natural method of family planning at five clinic sites in four cities, that is, Atlanta, Madison, Milwaukee, and Saint Louis, were invited to participate in a study that included use of a new electronic fertility monitor. The criteria for inclusion were the female partner must be between the age of 18 and 42; have a

\section{Fehring, Schneider, Raviele, \& Barron}


menstrual cycle range of 21 to 42 days; have not used depo-medroxyprogesterone acetate over the past 6 months; have no history of hormonal contraceptives for the past 3 months; if postbreastfeeding, have experienced at least three cycles past weaning; have no known fertility problems; not be using medications that interfere with ovulatory function; and not be pregnant. Male partners were to have no known fertility problem and be between the ages of 18 and 50 . All participants were to have the intent to avoid pregnancy for 12 months.

Of the 225 potential participants who sought clinical services in NFP and met the above criteria, 25 were provided initial instructions and a free fertility monitor but never produced data and were lost to follow-up, another 5 preferred to use a natural method without the monitor. The remaining 195 couples contributed data to the study from 2000 to 2005 . These 195 couples were enrolled in the study by convenience as they sought services at the five clinical sites. They were not randomly selected from a larger clinic population.

Mean age of the 195 female participants was 29.5 years ( $S D=5.9$, range 19-42) and the male partners 31.1 years $(S D=6.2$, range 18-49). The participants (expressed as female/male ratios) were primarily Caucasian (85/87\%), Catholic (87/81\%), at least high school educated $(91 / 90 \%)$, had a mean of 1.4 children $(S D=1.8$, range $0-8)$, and had combined household incomes greater than $\$ 50,000$. Approximately $7 \%$ of the couples were Hispanic.

The protocol for this study was approved by two Universities and one hospital-based internal review board for human rights compliance. The two private clinical sites provided letters of human rights approval from the directors of the clinics. All participants signed a consent form that explained the study and the request to use the NFP method for 12 months to avoid pregnancy. Each couple was provided with a free electronic fertility monitor to keep after participating in the study and was used as an incentive for participating. They were informed that if they wished to drop out of the study to achieve a pregnancy before the 12-month study requirement, they were to record their desire to achieve on their fertility chart.

\section{Measures}

The measures for this study included the CEFM and a fertility charting system for rating and graphing cervical mucus and for recording acts of intercourse. The CEFM is designed to detect a threshold level of urinary E3G and LH based on hormonal immunoassay techniques. Product testing has shown the monitor to be $98.8 \%$ accurate in detecting the LH surge in 169 of 171 cycles from 88 women, in agreement with a quantitative radioimmunoassay for $\mathrm{LH}$ (Fehring et al., 2004). In a study with 90 women who used the CEFM for 1 to 4 cycles, in 352 cycles with an LH surge, the first day of high fertility (i.e., the day of the first rise in E3G) was $3.01 \pm 2.33$

\section{Fehring, Schneider, Raviele, \& Barron}


days before the LH surge (May, 2001 ; Unipath Diagnostics, 2001).

The CEFM is initiated when a button on the monitor labeled " $M$ " is pushed on the first day of menses (see Figure 1). The monitor keeps track of the cycle day and requests either 10 or 20 daily urine tests per cycle. When the monitor requests a test, a test strip saturated with the women's urine is clipped into the monitor. The monitor will automatically scan the strip by a red laser light and report a low, high, or peak fertility reading on a small monitor screen. A high reading is provided when the threshold of E3G is reached and the peak reading when the threshold of LH is detected. Data from the CEFM can be downloaded into a computer and a display screen will provide length of the cycle, the day a test was requested, the day a test was performed, and the days of low, high, and peak fertility.

The charting system utilized in the study has room for recording the fertility level provided by the monitor and a 1 to 8 rating of the self-observed cervical mucus with the 1 mucus rating $=$ no mucus, no sensation; to the 8 rating = clear, slippery, stretchy mucus. The 1 to 8 rating allowed for a graphical and visual record of the mucus cycles and the estimated fertile window. The mucus rating system was developed by a reproductive endocrinologist (Dr. James Brown) and modified by Flynn and Lynch (1976). The charting system also has a place to record pregnancy intention for each menstrual cycle and a row for recording acts of intercourse (see Figure 2 for example fertility chart and cervical mucus rating system).

\section{Procedure}

The participating health professionals (i.e., two physicians and six professional nurses) from the five sites were experienced NFP teachers and either specialized in obstetrics, maternal child nursing, or women 's health. They all had completed an extensive training program in another system of NFP and had been teaching couples NFP from 5 to 15 years. All attended a 3-day workshop on how to teach a new standardized system of NFP with use of the CBFM and self-observation of cervical mucus. The new NFP system included three 1-hour group teaching sessions, and individual follow-ups at 1,2, and 6 months of use (or more if needed). The system of NFP has a standardized user manual, charting system, and documentation forms. No new health professionals were added to the study during the 5 years of data collection. The six health professionals were in constant contact through phone calls and e-mail.

All participants were asked to send their completed fertility charts to their NFP provider. In addition, they were to record on their fertility charts, before each new cycle of charting, their intention of either achieving or avoiding pregnancy. The NFP providers at the five sites collected copies of the fertility charts at the time of follow-ups and every 2 to 3 months until the participant

\section{Fehring, Schneider, Raviele, \& Barron}


completed the study, became pregnant, or dropped out of the study. All completed charts were sent to the research coordinators at Marquette University.

The rules for the method were that the couple user was to avoid intercourse and genital contact during the estimated fertile phase. The estimated fertile phase begins on the first day of the high reading on the monitor or the first observation of cervical mucus, whatever comes first. The end of the fertile phase was the last peak reading on the monitor or the last peak day of cervical mucus, whatever comes last, plus 3 full days.

\section{Classification of Pregnancy}

All participants with an intended or unintended pregnancy were interviewed by a health professional NFP teacher, had their fertility chart reviewed, and data downloaded from their fertility monitor. Each pregnancy was confirmed by a pregnancy urine test kit, blood test, or physician exam, or all. Pregnancies were classified as intentional only when a couple reported, prior to the pregnancy cycle, an intention to use the method to become pregnant. All unintentional pregnancies were classified as correct use when the rules of the methods were consistently followed. All unintentional pregnancies that occurred when the rules of the method were not always followed were classified as imperfect use. The total pregnancy rate was determined by the sum of correct- and imperfect-use unintended pregnancies (Lamprecht \& Trussell, 1997). Data from the charts were entered into the Statistical Package for the Social Sciences (SPSS, version 14; SPSS, Inc., Chicago, IL). Life table survival analysis was used to determine the correct-use and total (typical use) pregnancy rates. Based on a maximum pregnancy rate of 25 per 100 women over 12 months of use, a sample size of 132 to 220 participants was projected (Norman \& Steiner, 2000).

\section{Results}

Statistical characteristics of the menstrual cycles produced by this study can be found in a recent study on menstrual cycle variability (Fehring, Schneider, \& Raviele, 2006).

\section{Unintended Pregnancy Rates}

There was a total of 26 unintended pregnancies, of which 3 occurred with correct use, and a total of 1,795 documented months of use. Based on life table analysis, $97.9 \%$ (95\% confidence interval $[\mathrm{Cl}]=0.96-1.00$ ) of the participants did not have an unintended pregnancy over 12 months of correct use of the NFP method, that is, an unintended pregnancy rate of 2.0 per 100 women over 12 months of use. The imperfect-use 12-month unintended pregnancy rate (based on all unintended pregnancies and total months of use) was $85.8 \%(95 \% \mathrm{Cl}=0.82$ -

\section{Fehring, Schneider, Raviele, \& Barron}


0.94). In other words, there was a total (or imperfect use) unintended pregnancy rate of 14.2 per 100 women over 12 months of use (see Table 1 for a breakdown of unintended pregnancies by correct and imperfect use at 3-month intervals).

\section{Discontinuation}

The 195 participants contributed a mean of 9.2 months of use $(S D=3.8)$ and 102 $(52.3 \%)$ remained in the study for the agreed 12 months. Of the remaining 93 participants, 30 (15.4\%) were lost to follow-up, 19 (9.7\%) left to achieve a pregnancy, 18 (9.1\%) for personal reasons, and $3(1.5 \%)$ for health reasons. Of the 19 participants who intended to achieve a pregnancy, 12 did so in the first cycle of trying.

\section{Discussion}

This is the first report of an efficacy study in which the CEFM plus cervical mucus observations were used as a means to avoid pregnancy. The combined use of an electronic hormonal fertility monitoring plus cervical mucus observations as a method of NFP to avoid pregnancy revealed a correct-use unintended pregnancy rate of only 2.1 per 100 women over a 12-month trial. However, the total unintended pregnancy rate of 14.2 per 100 women over 12 months when the rules of the method were not always followed was much higher. The correctuse unintended pregnancy rate for this combined hormonal-mucus method is similar to the unintended pregnancy rate for periodic abstinence methods as reported by Trussell (2004). The total or imperfect-use unintended pregnancy rate of 14.2, however, is much lower than the unintended pregnancy rate of 25 per 100 women users over 12 months in the same report. The objectivity and accuracy of the fertility monitor could have contributed to the low correct-use rate as well as using two natural markers to estimate the fertile window.

Comparing unintended pregnancy rates for different methods of NFP pregnancy is difficult. There are many reasons for this difficulty including the differences in the systems of NFP, the different populations studied, the methods used to interpret pregnancies, the frequency of intercourse among the users, the age and education of the users, the various reproductive categories represented, the teaching methods and preparation of the NFP providers, and biases of the researchers (to name some obvious variables that could affect pregnancy rates) (Burkman, 2002; Trussell, 2004). However, comparison of studies with similar methods of NFP and similar participants can give the practitioner, the scientist, and the user information on relative efficacy.

In comparison with an earlier mucus observation-only NFP method efficacy study at

\section{Fehring, Schneider, Raviele, \& Barron}


Marquette University, the correct-use unintended pregnancy rate found in the current study was about the same as the earlier study, that is, a 12-month unintended pregnancy rate of 2.0 per 100 women users (Fehring et al., 1994). The 1994 Fehring et al. study did not report a total unintended pregnancy rate, so that comparison could not be made. However, Howard and Stanford (1999) found a total pregnancy rate of 17 per 100 users with the same CrM NFP method as the earlier Marquette study. The total pregnancy rate in the Howard and Stanford study is somewhat higher than the unintended pregnancy rate of 14.2 per 100 users in the current study. This comparison makes one wonder if adding the hormonal monitor does increase effectiveness of mucus observation - only method and thus is worth the expense and effort.

In comparison with the correct-use unintended pregnancy rate of 12 per 100 women over 13 cycles of use with the prototype Persona electronic hormonal fertility monitor, the correct-use unintended pregnancy rate of only 2.1 per 100 women users in the current study is much lower ( Bonnar et al., 1999 ). The addition of the cervical mucus observations with the CBFM serves as a double check for both the beginning and end of the fertile phase and seems to enhance the ability to estimate the fertile phase with more accuracy. This makes sense since the CEFM and the prototype Persona monitor often underestimates the beginning of the fertile window. A double check for the beginning and end of the fertile window with cervical mucus plus temperature was found to work well for the double-check NFP method developed for German women (Frank-Herrmann et al., 2005).

\section{Limitations}

Since the current study was not a comparison study, the results could be influenced by many factors, including biases of the researchers and clinicians contributing to the study (Grimes, Gallo, Grigorieva, Nanda, \& Schulz, 2005). Another limitation is use of two markers of fertility at times confused the participants, for example, when there was low reading on the monitor and cervical mucus present, or when the peak reading with the monitor did not correlate with the peak in cervical mucus. Having two markers of fertility also compounds the teaching and complexity of a fertility awareness - based method.

A difficulty in the study was finding participants to agree to avoid pregnancy for 12 months. Many of the young couples either dropped out prematurely or started to test the ends of the estimated fertile window without declaring that they are now trying to achieve a pregnancy. The decision to achieve a pregnancy does not always begin with a 100\% effort (Howard \&

\section{Fehring, Schneider, Raviele, \& Barron}


Stanford, 1999). Unintended intercourse patterns reveal there is often a first testing of the ends of the estimated fertile phase. The intercourse patterns found in this study tend to show that those unintended pregnancies occurred at the ends and just outside of the end of the estimated fertile phase (see Figure 3 in published version). Another explanation for testing the ends of the fertile phase, however, might be that the couples were tired of the length of periodic abstinence. Although the frequency of intercourse, among the participants, was similar to previous studies, there was an underreporting of intercourse during the fertile time. This was apparent when a few participants would reveal this several months after the pregnancy interview. The average frequency of intercourse per cycle in the current study was 3.85 ( $S D=3.32$, range 1-24), but many of the cycles $(16 \%)$ had missing data or no recorded intercourse due to uneasiness of sharing that information.

\section{Implication for Practicing Nurses}

Professional nurses, advanced practice nurses, and other health professionals who teach couples methods of NFP could easily integrate the use of the CEFM with current methods of NFP. If they are teaching a cervical mucus - only NFP method, the CEFM readings could serve as a second check for the beginning and end of the fertile window and help to clarify the fertile phase when mucus observations are confusing. Those nurses and health professionals who teach a combined mucus and temperature method could have couples drop the use of temperature as a second check for the fertile phase and substitute the CEFM readings or they could add the CEFM as another marker. One of the simplest methods would be to combine the CEFM readings with a calendar rhythm formula. A simple algorithm has been developed for use

of the monitor alone that serves as a double check for the beginning and end of the fertile phase (see Fehring, 2005). Simplifying the use of the monitor as a means to avoid pregnancy would increase the ability of health professionals to provide this method in a short office visit and make it easier to learn and to use.

However, when nurses and other health professionals teach the use of the monitor along with other markers of fertility, they need to emphasize when using both cervical mucus (or BBT) monitoring plus the CEFM to be consistent in monitoring both markers and avoiding intercourse when either indicates a fertile day. Waiting 3 full days after the peak in the monitor or mucus is recommended, that is, resuming intercourse on the evening of the fourth day past either peak.

\section{Research Recommendations}

There are few good randomized control trials with NFP methods (Grimes et al., 2005). A recommended research effort would be to conduct a randomized control trial of using the CEFM 12 Fehring, Schneider, Raviele, \& Barron 
plus a simple calendar formula in comparison with cervical mucus monitoring plus a simple calendar formula as a double check for the beginning and end of the estimated fertile phase. The use of the CBFM along with cervical mucus observations or with a simple fertility formula also needs to be tested with more varied populations, especially Hispanic and African American populations. Furthermore, efficacy of the CBFM needs to be tested with women with special reproductive categories, including breastfeeding and perimenopause.

\section{Conclusions}

In conclusion, the use of an electronic hormonal fertility monitor that was designed for couples to achieve pregnancy, when used along with another marker of fertility, can be effective as or more effective than current NFP methods. Designing simpler protocols for using the monitor for monitoring fertility for special circumstances such as breastfeeding and not ovulating is in progress (Fehring, Schneider, \& Barron, 2005).

\section{Acknowledgments}

The authors thank the following individuals for data charts: Susana Crespo, RN, Julie Krause, RN, Peggy Mc-Intyre, RN, Cynthia Jones-Nosacek, MD, and Susan Lepak.

\section{References}

Barron, M . L ., \& Fehring, R . (2005). Basal body temperature assessment: Is it useful for couples seeking pregnancy. Maternal Child Nursing, 30, 290-296.

Behre, H. M., Kuhlage, J., Gassner, C., Sonntag, B., Schem, C., Schneider, H. P. G., et al. (2000). Prediction of ovulation by urinary hormone measurements with the home use Clearplan Fertility Monitor: Comparison with transvaginal ultrasound scans and serum hormone measurements. Human Reproduction, 15, 2478-2482.

Bonnar, J., Flynn, A., Freundl, G., Kirkman, R., Royston, R., \& Snowden, R. (1999). Personal hormone monitoring for contraception. British Journal of Family Planning, 24, 128-134.

Burkman, R. T. (2002). Clinical pearls: Factors affecting reported contraceptive efficacy rates in clinical studies. International Journal of Fertility, 47, 153-161.

Colombo, B., Mion, A., Passarin, K., \& Scarpa, B. (2006). Cervical mucus symptom and daily fecundability: First results from a new database. Statistical Methods in Medical Re-

\section{Fehring, Schneider, Raviele, \& Barron}


search, 15, 161-180.

Crosignani, P. G., \& Rubin, B. L. (2000). Optimal use of infertility diagnostic tests and treatments. Human Reproduction, 15, 723-732.

Dunson, D. B., Baird, D. D., Wilcox, A. J., \& Weinberg, C. R. (1999). Day-specific probabilities of clinical pregnancy based on two studies with imperfect measures of ovulation. Human Reproduction, 14, 1835-1839.

Fehring, R. J. (1990). Methods used to predict ovulation, a comparative study. Journal of Obstetric, Gynecological, and Neonatal Nursing, 19, 233-237.

Fehring, R. J. (2002). Accuracy of the peak day of cervical mucus as a biological marker of fertility. Contraception, 66, 231-235.

Fehring, R. J. (2005). New low and high tech calendar methods of family planning. Journal of Nurse Midwifery and Women's Health, 50, 31-38.

Fehring, R. J., Lawrence, D., \& Philpot, C. (1994). Use effectiveness of the Creighton model ovulation method of natural family planning. Journal of Obstetric, Gynecologic, and Neonatal Nursing, 23, 303-312.

Fehring, R. J., Raviele, K., \& Schneider, M. (2004). A comparison of the fertile phase as determined by the Clearplan Easy Fertility Monitor ${ }^{\mathrm{TM}}$ and self-assessment of cervical mucus. Contraception, 69, 9-14.

Fehring, R. J., Schneider, M., \& Barron, M. (2005). Protocol for determining fertility while breastfeeding. Fertility and Sterility, 84, 805-807.

Fehring, R. J., Schneider, M., \& Raviele, K. (2006). Variability in the phases of the menstrual cycle. Journal of Obstetric, Gynecologic, and Neonatal Nursing, 35, 376-384.

Flynn, A. M., \& Lynch, S. S. (1976). Cervical mucus and identification of the fertile phase of the menstrual cycle. British Journal of Obstetrics and Gynecology, 83, 656-659.

Frank-Herrmann, P., Gnoth, C., Baure, S., Strowitski, T., \& Freundl, G. (2005). Determination of the fertile window: Reproductive competence of women - European cycle databases. Gynecology and Endocrinology, 20, 305-312.

Grimes, D. A., Gallo, M. F., Grigorieva, V., Nanda, K., \& Schulz, K. F. (2005). Fertility awareness-based methods for contraception: Systematic review of randomized controlled trials. Contraception, 72, 85-90.

Guida, M., Tommaselli, G. A., Palomba, S., Pellicano, M., Moccia, G., DiCarlo, C., et al. (1999). Efficacy of methods for determining ovulation in a natural family planning program. Fertility and Sterility, 72, 900-904.

\section{Fehring, Schneider, Raviele, \& Barron}


Howard, M. P., \& Stanford, J. B. (1999). Pregnancy probabilities during use of the Creighton model fertility care system. Archives of Family Medicine, 8, 391-402.

Kambic, R. T., \& Lamprecht, V. (1996). Calendar rhythm efficacy: A review. Advances in Contraception, 12, 123-128.

Lamprecht, V., \& Trussell, J. (1997). Effectiveness studies on natural methods of natural family planning. Advances in Contraception, 13, 155-165.

Martinez, A. R. (1997). Prediction and detection of the fertile phase of the menstrual cycle: An overview. Advances in Contraception, 13, 131-138.

Martinez, A. R., Zinaman, M. J., Jennings, V. H., \& Lamprecht, V. M. (1995). Prediction and detection of the fertile period: The markers. International Journal of Fertility, 40, 139-155.

May, K. (2001). Home monitoring with the ClearPlan Easy Fertility Monitor for fertility awareness. Journal of International Medical Research, 29(Suppl. 1), 14A-20A.

McCarthy, J. J., \& Rockette, H. F. (1983). A comparison of methods to interpret the basal body temperature graph. Fertility and Sterility, 39, 640-646.

Mosher, W. D., Martinez, G. M., Chandra, A., Abma, J., \& Wilson, S. J. (2004). Use of contraception and use of family planning services in the United States: 1982-2002. Advance Data, 10, 1-36.

Norman, R. G., \& Steiner, D. L. (2000). Biostatistics: The bare essentials. Hamilton, Ontario: B.C. Decker.

Nulsen, J., Wheeler, C., Ausmanas, M., \& Blasco, L.(1987). Cervical mucus changes in relationship to urinary luteinizing hormone. Fertility and Sterility, 48, 783-786.

Quagliarello, J., \& Arny, M. (1986). Inaccuracy of basal body temperature charts in predicting urinary luteinizing hormone surges. Fertility and Sterility, 5, 334-337.

Robinson, J. E., Wakelin, M., \& Ellis, J. E. (2007). Increased pregnancy rate with use of the Clearblue Easy Fertility Monitor. Fertility and Sterility, 87, 329-334.

Seibel, M. (1986). Luteinizing hormone and ovulation timing. Journal of Reproductive Medicine, 31(Suppl.), 754-759.

Stanford, J. B., White, G. L., \& Hataska, H. (2002). Timing intercourse to achieve pregnancy: Current evidence. Obstetrics \& Gynecology, 100, 1333-1341.

Tanabe, K., Susumu, N., Hand, K., Nishii, K., Ishikawa, I., \& Nozawa, S. (2001). Prediction of the potentially fertile period by urinary hormone measurements using a new home-use monitor: Comparison with laboratory hormone analyses. Human Reproduction, 16,

\section{Fehring, Schneider, Raviele, \& Barron}


1619-1624.

Trussell, J. (2004). Contraceptive failure in the United States. Contraception, 70, 89-96.

Trussell, J., \& Grummer-Strawn, L. (1991). Further analysis of contraceptive failure of the ovulation method. American Journal of Obstetrics and Gynecology, 165, 2054-2059.

Unipath Diagnostics. (2001). Professional information: Clear-plan easy fertility monitor. Princeton, NJ: Author.

Wilcox, A. J., Weinberg, C. R., \& Baird, D. D. (1995). Timing of sexual intercourse in relation to ovulation; effects of the probability of conception, survival of the pregnancy, and sex of the baby. New England Journal of Medicine, 333, 1517-1521.

World Health Organization Task Force. (1981). A prospective multicentre trial of the ovulation method of natural family planning. II. The effectiveness phase. Fertility and Sterility, 36, 591-598.

\section{Appendix}

\section{Figure 1}

Clearblue Easy Fertility Monitor.

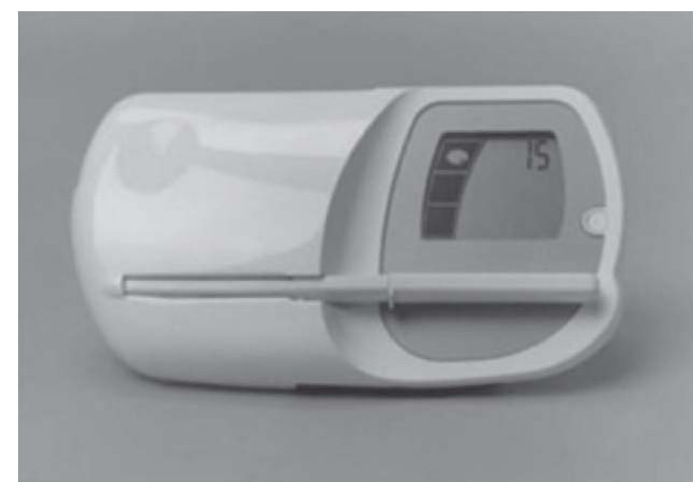




\section{Figure 2}

Fertility charting system with rating and graphing of cervical mucus observations on 1 to 8 scale.

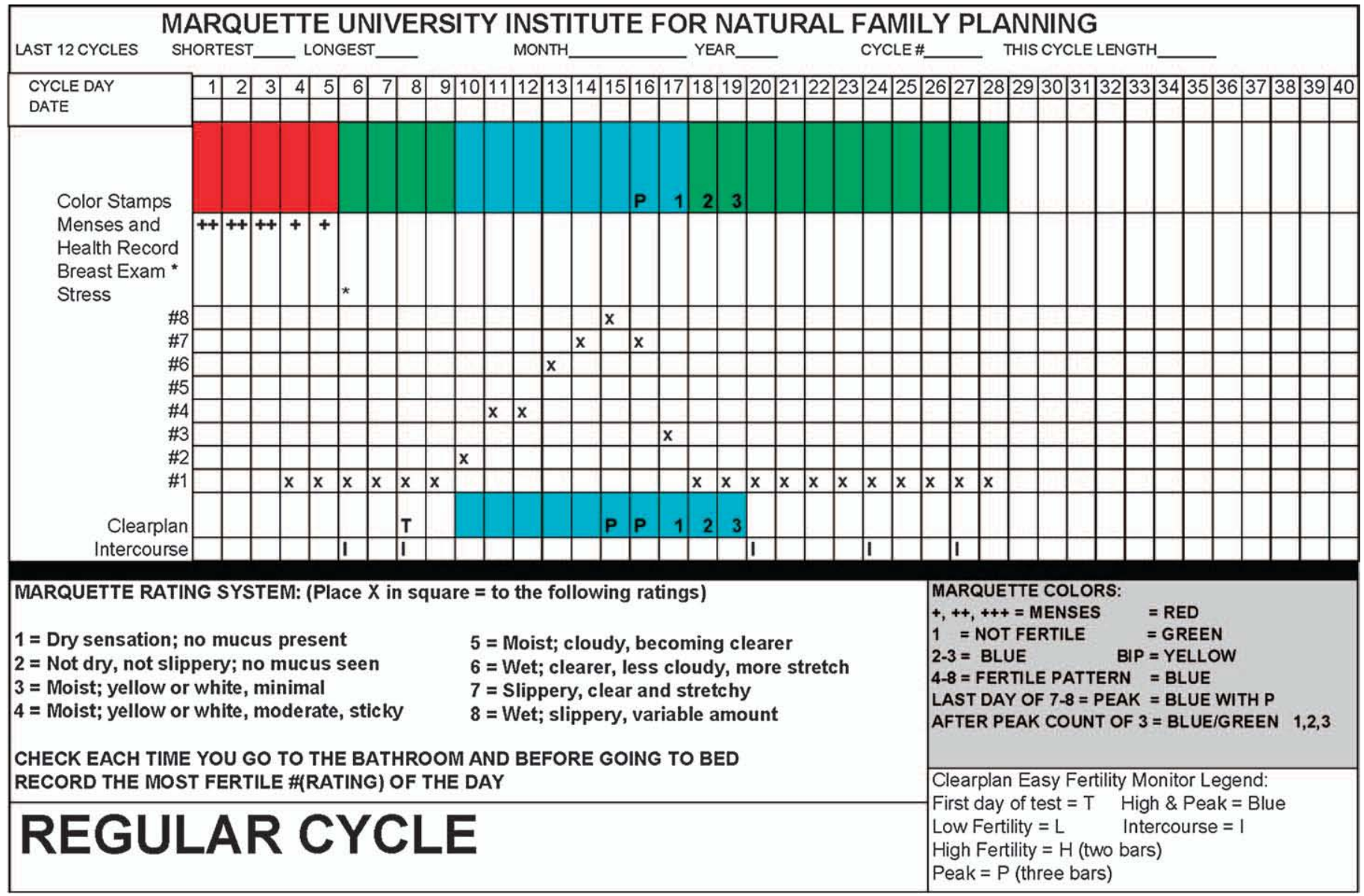




\section{Table 1}

Twelve-Month Correct and Typical Effectiveness Rates in Avoiding Pregnancy with use of the Clearblue Easy Fertility Monitor Plus Cervical Mucus Monitoring ( $\mathrm{N}=195)$

\begin{tabular}{|c|c|c|c|c|}
\hline Months of Use & No. of Pregnancies & Correct use & No. of Pregnancies & Typical Use \\
\hline 3 & 1 & 0.994 & 8 & 0.958 \\
\hline 6 & 0 & 0.994 & 6 & 0.922 \\
\hline 9 & 1 & 0.987 & 5 & 0.890 \\
\hline 12 & 1 & 0.979 & 4 & 0.858 \\
\hline Total & 3 & & 23 & \\
\hline
\end{tabular}

